\title{
Health professionals' views on the barriers and enablers to evidence-based practice for acute stroke care: a systematic review
}

Leonard Baatiema ${ }^{1,2^{*}}$ (D) Michael E. Otim³ ${ }^{3}$ George Mnatzaganian ${ }^{4}$, Ama de-Graft Aikins ${ }^{1}$, Judith Coombes ${ }^{5}$ and Shawn Somerset ${ }^{2}$

\begin{abstract}
Background: Adoption of contemporary evidence-based guidelines for acute stroke management is often delayed due to a range of key enablers and barriers. Recent reviews on such barriers focus mainly on specific acute stroke therapies or generalised stroke care guidelines. This review examined the overall barriers and enablers, as perceived by health professionals which affect how evidence-based practice guidelines (stroke unit care, thrombolysis administration, aspirin usage and decompressive surgery) for acute stroke care are adopted in hospital settings.

Methodology: A systematic search of databases was conducted using MEDLINE, Cumulative Index to Nursing and Allied Health Literature (CINAHL), Embase, PsycINFO, Cochrane Library and AMED (Allied and Complementary Medicine Database from 1990 to 2016. The population of interest included health professionals working clinically or in roles responsible for acute stroke care. There were no restrictions to the study designs. A quality appraisal tool for qualitative studies by the Joanna Briggs Institute and another for quantitative studies by the Centre for Evidence-Based Management were used in the present study. A recent checklist to classify barriers and enablers to health professionals' adherence to evidence-based practice was also used.
\end{abstract}

Results: Ten studies met the inclusion criteria out of a total of 9832 search results. The main barriers or enablers identified included poor organisational or institutional level support, health professionals' limited skills or competence to use a particular therapy, low level of awareness, familiarity or confidence in the effectiveness of a particular evidence-based therapy, limited medical facilities to support evidence uptake, inadequate peer support among health professionals', complex nature of some stroke care therapies or guidelines and patient level barriers.

Conclusions: Despite considerable evidence supporting various specific therapies for stroke care, uptake of these therapies is compromised by barriers across organisational, patients, guideline interventions and health professionals' domains. As a result, we recommend that future interventions and health policy directions should be informed by these findings in order to optimise uptake of best practice acute stroke care. Further studies from low- to middle-income countries are needed to understand the barriers and enablers in such settings.

Trial registration: The review protocol was registered in the international prospective register of systematic reviews, PROSPERO 2015 (Registration Number: CRD42015023481)

Keywords: Acute stroke, Evidence-based practice, Therapies, Services, Barriers, Enablers

\footnotetext{
* Correspondence: leonard.baatiema@acu.edu.au

'Regional Institute for Population Studies, University of Ghana, P.O Box LG96,

Legon-Accra, Ghana

${ }^{2}$ School of Allied Health, Faculty of Health Sciences, Australian Catholic

University, Sydney, Australia

Full list of author information is available at the end of the article
} 


\section{Background}

Translation of research evidence into clinical practice is a major imperative for health professionals in policy, management and research, worldwide. It is almost half a century since Cochrane challenged conventional health care practices which consequently paved the way for the present day evidence-based practice movement in medicine and health care [1]. Nonetheless, routine clinical practice still lags behind contemporary research evidence [2-4], despite international calls for research evidence to guide healthcare delivery [5]. Globally, there is no single solution to closing this knowledge to practice gap [4, 6, 7]. In general, it has been estimated to take about 17 years for research evidence to be translated into clinical practice [8]. Delays in the adoption of evidence-based practice could be attributed to a multiplicity of barriers [9-12] underpinned by a plethora of theoretical and conceptual perspectives [2, 3, 13, 14], which have emerged to shed light on these barriers or enablers.

Stroke is caused by an interruption or blockage in blood supply or arterial bleeding into or around the brain $[15,16]$. The early stages (first $48 \mathrm{~h}$ ) of an acute stroke are a critical time-window for appropriate interventions to either stop or slow down brain tissue decay and minimise mortality and morbidity [17]. To provide acute stroke care in the early stages, current recommendations from Level-1 evidence for best practice include (1) stroke care in a specialist stroke unit [18, 19], (2) thrombolytic therapy with intravenous tissue plasminogen activator (t-PA) within $4.5 \mathrm{~h}$ of an acute ischemic stroke [20-22], (3) aspirin administration within $48 \mathrm{~h}$ of acute ischemic stroke onset [23-25] and (4) decompressive surgery if required within $48 \mathrm{~h}$ of stroke onset $[26,27]$. The use of t-PA for example is the most effective pharmacological therapy for acute ischemic stroke despite the persistence of controversies surrounding its usage. First reported by the National Institute of Neurological Disorders and Stroke (NINDS) trials with a treatment window of $3 \mathrm{~h}$ [22], a later trial extended the treatment time to $4.5 \mathrm{~h}$ upon acute ischemic stroke [21]. Given such evidence consistently showed sound clinical outcomes over time, clinical guidelines have been developed and continually updated to support the application of these interventions for improved patient outcomes [28-34]. However, despite this scientific evidence and increased support for their usage, translation into clinical practice is slow, and this is greatest in low-middle income countries [35-37]. For example, despite the net benefits associated with thrombolytic therapy for acute ischemic stroke, global uptake in low-income countries is about $3 \%$ compared to $50 \%$ in high-income countries [35]. A recent survey of acute stroke services in eleven major referral hospitals in Ghana also revealed the lack of use of t-PA for acute ischemic stroke care and the availability of only one stroke unit [38].

The reasons for the slow uptake remains poorly understood. Some studies have however attempted to shed light on such barriers, and these comprised inadequate medical facilities for acute stroke care, health professionals' unwillingness for change, unawareness of evidence-based therapies, lack of health professionals' competence to apply evidence-based therapies, limited staff capacity and decision-makers' values and preferences could be attributed for the slow uptake [39-46]. Such barriers have resulted in the underutilisation of best practice interventions towards positive clinical outcomes. The recent Lancet series on Right Care [47], which seeks among others, to highlight the chronic underutilization of evidence-based interventions further underscores the centrality of this review.

To date, no study has attempted to systematically analyse published primary studies on the barriers and enablers perceived by health care professionals to influence the adoption of these four highly recommended acute stroke therapies or services. Prior studies on this topic were either limited in focus by only unilaterally exploring barriers related to the use of t-PA [41, 42, 44], neuroprotective therapy [45] or generalised acute stroke care guidelines [40]. A recent study by Craig et al. has also attempted to examine some of these barriers and enablers [48], though an important contribution, a different theoretical framework was used, and focused more on clinical behavioural components.

Our aim in this review was to identify health professionals' views on the barriers and enablers to their use of the above recommended evidence-based acute stroke care interventions. An understanding of these barriers and enablers is important towards closing the current knowledge to practice gap in stroke clinical practice. With the increasing stroke burden in low-middle income regions in recent times [49-51] and where uptake levels of such interventions are presently lowest, a clearer understanding of barriers and enablers, primarily from such regions may also be essential in developing context-specific strategies to optimise uptake of evidence-based acute stroke care recommendations in clinical practice to improve patient outcomes.

\section{Methods}

This review was conducted according to the Preferred Reporting Item for Systematic Reviews and MetaAnalysis (PRISMA) systematic review approach [52], as outlined in Additional file 1. The review protocol was registered in the international prospective register of systematic reviews, PROSPERO 2015 (Registration Number: CRD42015023481). 


\section{Eligibility criteria}

Studies based on the views of stroke specialists, medical doctors, nurses and allied health professionals were considered. Other health professionals including health managers, health planners, health policy-makers or any health executives' about barriers or enablers to the uptake of evidence-based acute stroke care were included. For inclusion, interventions for evidence-based acute stroke care were restricted to barriers or enablers in relation to the provision of care in a stroke unit, thrombolytic therapy, the use of aspirin and decompressive surgery. Peer-reviewed articles of any study design were considered. Barriers and enablers based on database records were excluded. Included studies were based only on the views, opinions and experiences of the health professionals. Non-original research such as letters, commentaries, guidelines, magazines and editorials were excluded. Research studies with non-human components were also not considered.

\section{Search strategy}

A systematic search of the literature was conducted electronically using MEDLINE, CINAHL, Embase, PsycINFO, Cochrane Library and AMED. Reference lists and bibliographies from eligible studies published from 1990 to 2016 were also considered for inclusion. The review considered studies published within this time duration to correspond with the period when evidencebased medicine movement and scholarship enjoyed renewed interest and acknowledgement [53]. This was also done to ensure included studies reflect current evidence of health professionals' views on what acts as a barrier or an enabler to their uptake or adherence to evidence-based practice for acute stroke care. Due to lack of resources for language translation, all included studies were limited to studies published in English language. Search strings were designed to reflect related Medical Subject Heading ( $\mathrm{MeSH}$ ) terms, key terms and phrases from the selected databases related to the review aim. Details of the search terms used are presented in Additional file 2.

\section{Study selection}

Results were downloaded and imported into EndNote for screening to first remove duplicates by one author (LB). The next stage involved the screening of the remaining studies based on the relevance of study titles and abstracts to the review aim. When articles had insufficient information in the title and abstract to support this screening, a full-text reading was conducted. This was followed by the selection of all potentially eligible studies in full text. A second author (SS) reviewed the selected full-text articles to ensure they met the eligibility criteria. Results of the full text were also shared with the remaining authors to validate, and none of the authors raised questions about their eligibility. Articles which met the inclusion criteria following full-text screening by two authors (LB and SS) were selected for the final analysis.

\section{Data extraction}

A standardised data extraction tool (evidence table) was used to extract information relevant to the study aim by one author (LB). As shown in Table 1, the information extracted include the authors and year of publication, country of study, intervention, study aim, design, participants/sample, data collection methods and key findings on the barriers and enablers to uptake of acute stroke care interventions. This was systematically done to ensure extracted data characteristics from the eligible studies were consistent. The key findings and conclusions of the eligible studies which were reported as either barriers, enablers or barriers and enablers were identified by one author (LB). These findings were shared with the remaining reviewers to ensure consistency with the primary studies.

\section{Data synthesis}

Data analysis involved a thematic analysis of the results from the eligible studies. Based on the Tailored Implementation for Chronic Diseases project [54], a preexisting framework of seven domains developed by implementation science researchers to examine what informs change in clinical practice [55] was followed to categorise the themes of barriers and enablers. The checklist of seven domains comprised guideline factors, individual health professionals' factors, patient factors, professional interactions, incentives and resources, capacity for organisational change, and social, political and legal affairs. Additional file 3 provides further explanation of each domain. This process was done by one reviewer (LB) who is experienced in categorising themes using pre-existing frameworks. This was done with constant reference to the content of the pre-existing framework and identified barriers and enablers from the articles to ensure appropriate classification. Another reviewer (SS) validated the classification of the barriers and enablers (See Table 2), and one disagreement was recorded during this stage but was quickly resolved in consultation with another author (AdGA). One author (LB) consequently weighted each domain of barriers/enablers in a tabular form (See Table 3) according to the frequency of each barrier or enabler as reported in the articles.

\section{Assessment of methodological quality}

To capture the unique reporting differences within qualitative and quantitative studies, two separate quality 


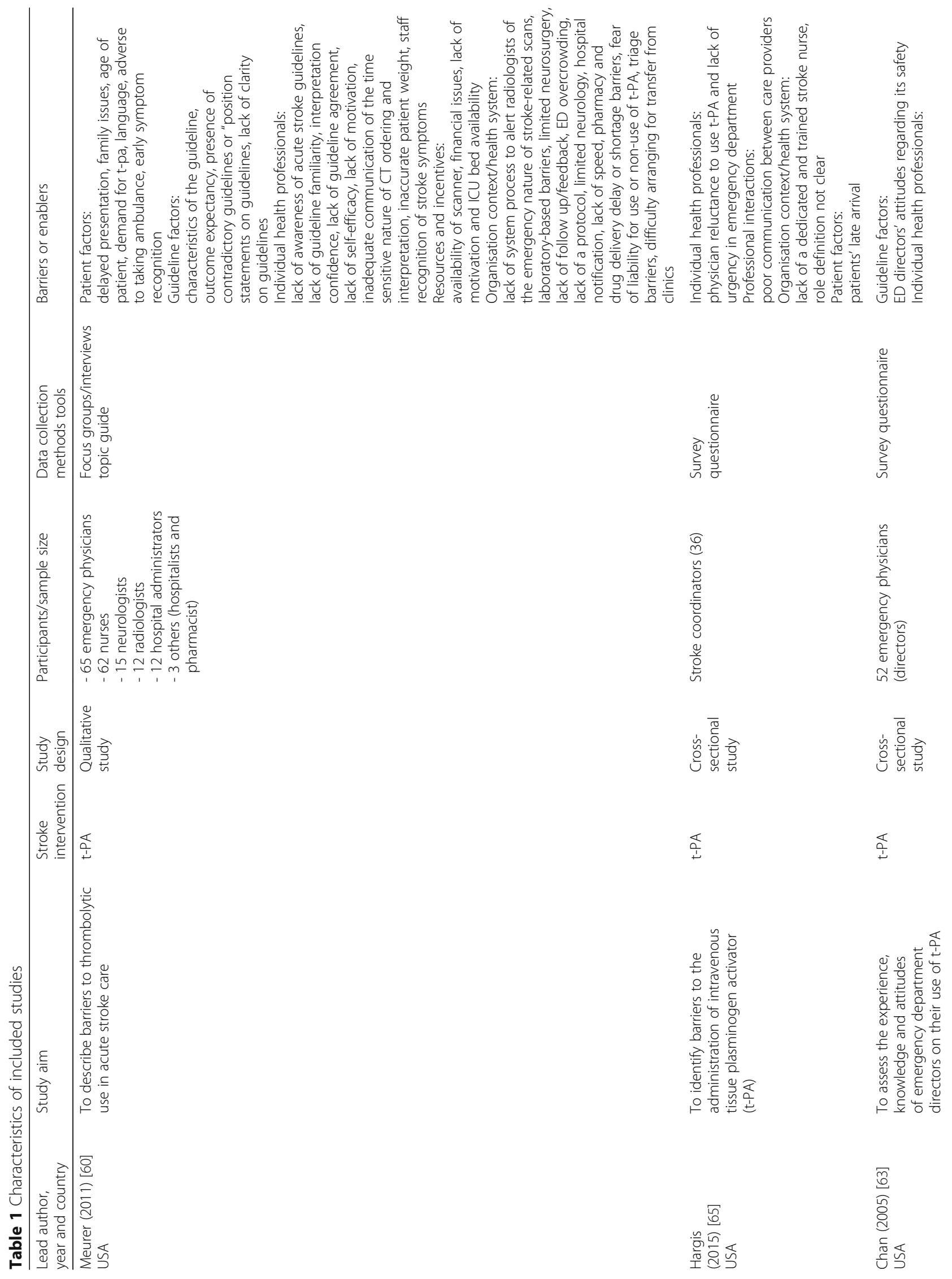




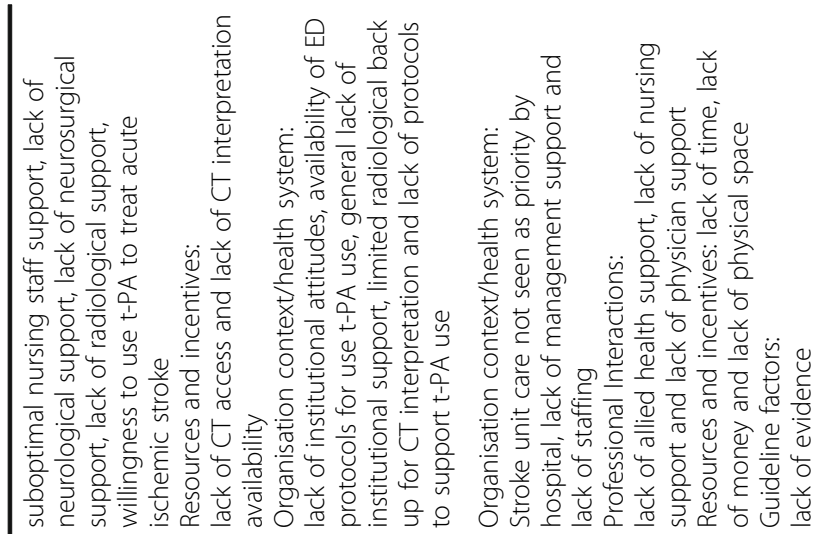

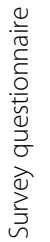

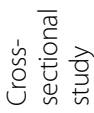

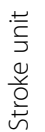

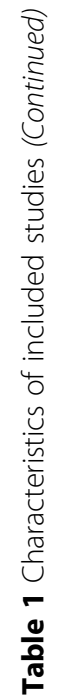

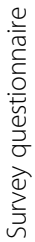
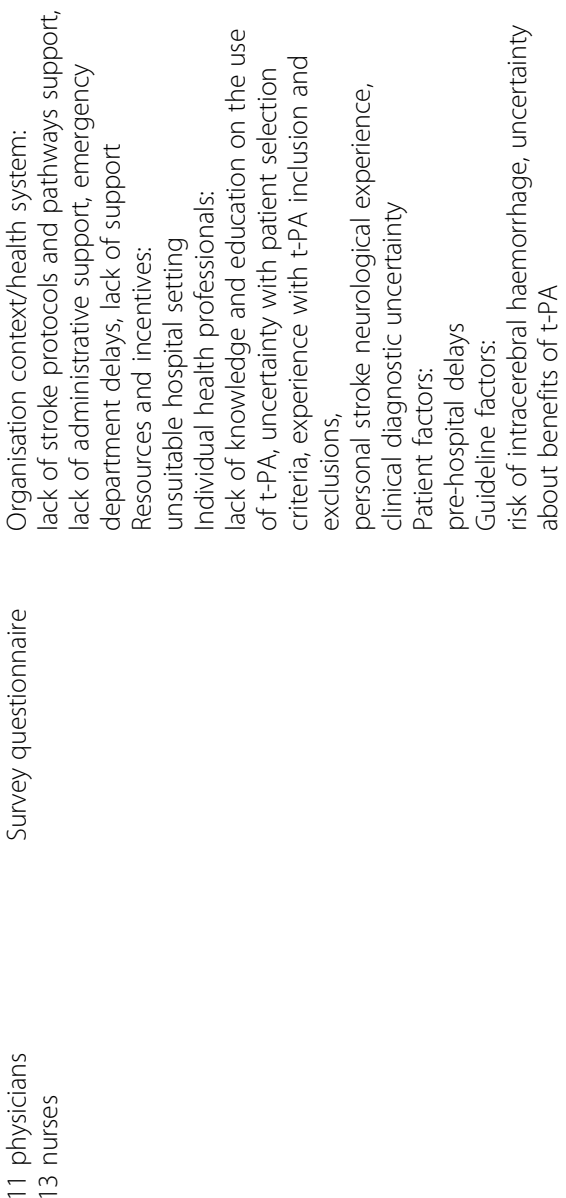

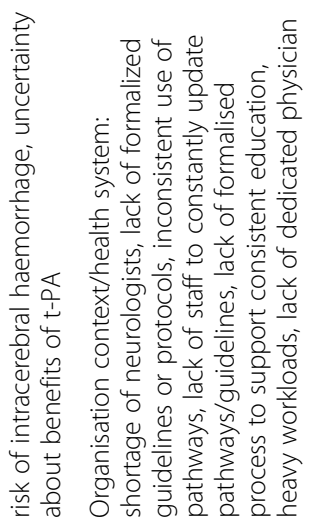

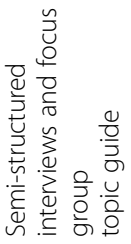

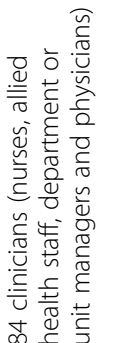

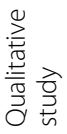

蒿焉

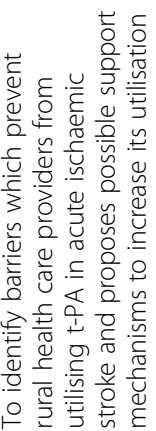

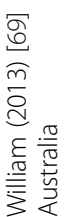

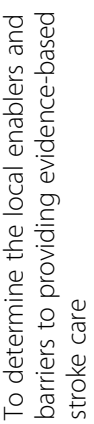

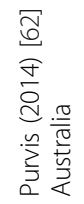




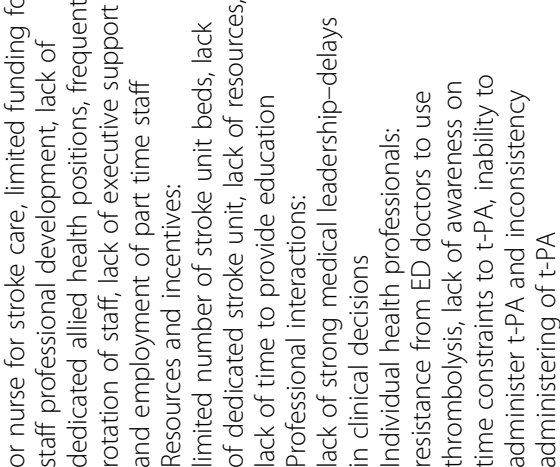




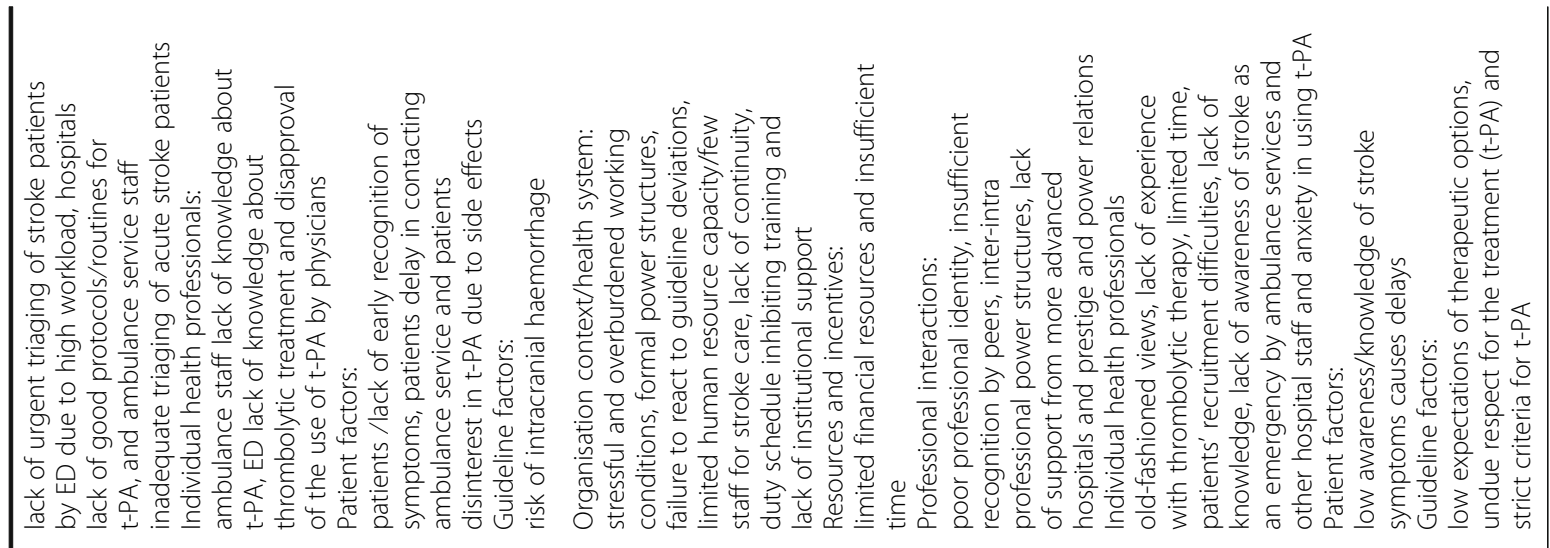

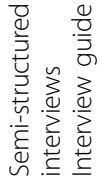

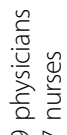

$\overline{\widetilde{\Sigma}}$

岕总总旁

总

$\stackrel{\square}{+}$

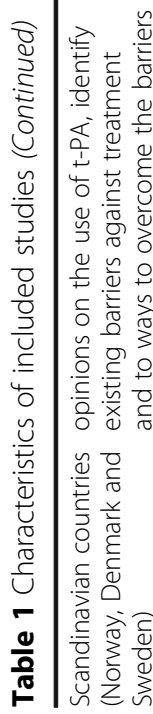

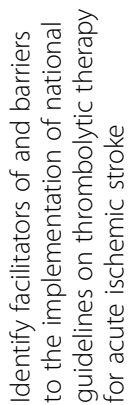

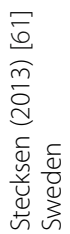


Table 2 Domain of barriers or enablers to evidence uptake

\begin{tabular}{|c|c|c|c|c|c|c|c|c|}
\hline Author and year & $\begin{array}{l}\text { Stroke therapy } \\
\text { or intervention }\end{array}$ & $\begin{array}{l}\text { Guideline } \\
\text { factors }\end{array}$ & $\begin{array}{l}\text { Individual health } \\
\text { professionals }\end{array}$ & $\begin{array}{l}\text { Patient } \\
\text { factors }\end{array}$ & $\begin{array}{l}\text { Professional } \\
\text { interactions }\end{array}$ & $\begin{array}{l}\text { Incentives and } \\
\text { resources }\end{array}$ & $\begin{array}{l}\text { Capacity for } \\
\text { organisational } \\
\text { change }\end{array}$ & $\begin{array}{l}\text { Social, political } \\
\text { and legal factors }\end{array}$ \\
\hline O'Rourke (2013) [66] & Stroke unit & $x$ & & & $x$ & $x$ & $x$ & \\
\hline Grady (2014) [64] & Thrombolysis & & $x$ & & $x$ & & $x$ & \\
\hline William (2013) [69] & Thrombolysis & $x$ & $x$ & $x$ & & & $x$ & \\
\hline Van Der (2004) [68] & Aspirin and thrombolysis & $x$ & $x$ & $x$ & & $x$ & $x$ & \\
\hline Slot (2009) [67] & Thrombolysis & $x$ & $x$ & $x$ & & $x$ & $x$ & \\
\hline Meurer (2011) [60] & Thrombolysis & $x$ & $x$ & $x$ & & $x$ & $x$ & \\
\hline Purvis (2014) [62] & $\begin{array}{l}\text { Stroke unit and } \\
\text { thrombolysis }\end{array}$ & $x$ & $x$ & $x$ & $x$ & $x$ & $x$ & \\
\hline Stecksén (2013) [61] & & $x$ & $x$ & & $x$ & $x$ & $x$ & \\
\hline Hargis (2015) [65] & Thrombolysis & $x$ & $x$ & $x$ & $x$ & $x$ & $x$ & \\
\hline Chan (2005) [63] & Thrombolysis & $x$ & $x$ & & & $x$ & $x$ & \\
\hline
\end{tabular}

$\mathrm{X}$ indicates a particular thematic barrier or enabler reported by the author (s)

reporting assessment tools were used. The checklist by the Joanna Briggs Institute for assessing qualitative studies was used for the qualitative studies [56], while the guidelines suggested by the Centre for Evidence-Based Management to appraise surveys was also used for the quantitative studies [57]. These checklists were used because they have comprehensively clear score sheets and instructions which enabled the authors to assess the relevance and rigour of all included studies. Given that there is still lack of consensus on the criteria for assessing the quality of qualitative studies in systematic reviews [58, 59]; included qualitative studies were not based on their quality scoring but on the basis of their overall contribution to the synthesis rigour. One reviewer (LB) appraised the quality of included studies. Another reviewer (SS) carried out a separate rating and slight variations were observed. However, these differences were quickly addressed by the two reviewers.

\section{Results}

\section{Study selection}

The electronic search yielded 9832 studies [MEDLINE $=2518$, $\mathrm{CINAHL}=458, \mathrm{AMED}=221, \mathrm{PscINFO}=1229$, Embase $=873$, Cochrane Library $=4507$ and 26 additional studies retrieved from other sources]. After removing 1386 duplicates, 8446 studies remained. Screening based on title and abstract relevance excluded 8263 and 81articles, respectively. Studies excluded at this stage were either due to the fact that they were not primary studies, had irrelevant topics, that is, not focused on barriers and enablers to the four recommended evidencebased stroke care interventions. Other reasons for exclusion include duplicate studies, letters and editorials. A full-text screening of the remaining 102 potentially eligible studies led to further exclusions of 92 studies as they were deemed irrelevant to the study aim, focused on different population of interest, included review papers, guidelines and case reports. Overall, 10 studies met the inclusion criteria (See Fig. 1).

\section{Study characteristics}

Three qualitative [60-62] and seven quantitative studies [63-69] were included. Quantitative studies employed online and postal surveys while the qualitative studies used semi-structured interviews and focus group methods. Whereas analysis of the quantitative studies was conducted using predominantly descriptive statistics, thematic analysis guided the analyses of the qualitative studies. The total number of included participants was 1692, and these comprised nurses, general medical doctors, neurologists, emergency department physicians, allied health staff and health managers. Included studies were published between 2004 and 2015. Four studies were conducted in Australia [62, 64, 66, 69], three in the USA [60, 63, 65], two in Sweden [61, 67], and one each in Norway [67], Denmark [67] and the Netherlands [68]. Most of the barriers or enablers identified in the quantitative studies were also found in the qualitative studies. Studies predominantly examined the barriers or enablers to the use of thrombolysis $[60,61,63-65,67,69]$. One study focused exclusively on barriers related to the establishment of a stroke unit [66], another on the uptake of both aspirin and thrombolysis [68] and the remaining on stroke unit and thrombolysis [62]. Although most of the eligible studies focused on barriers related to the use of evidence-based care for acute stroke, all included studies reported on three or more related barriers or enablers (See Table 1 for additional information). 


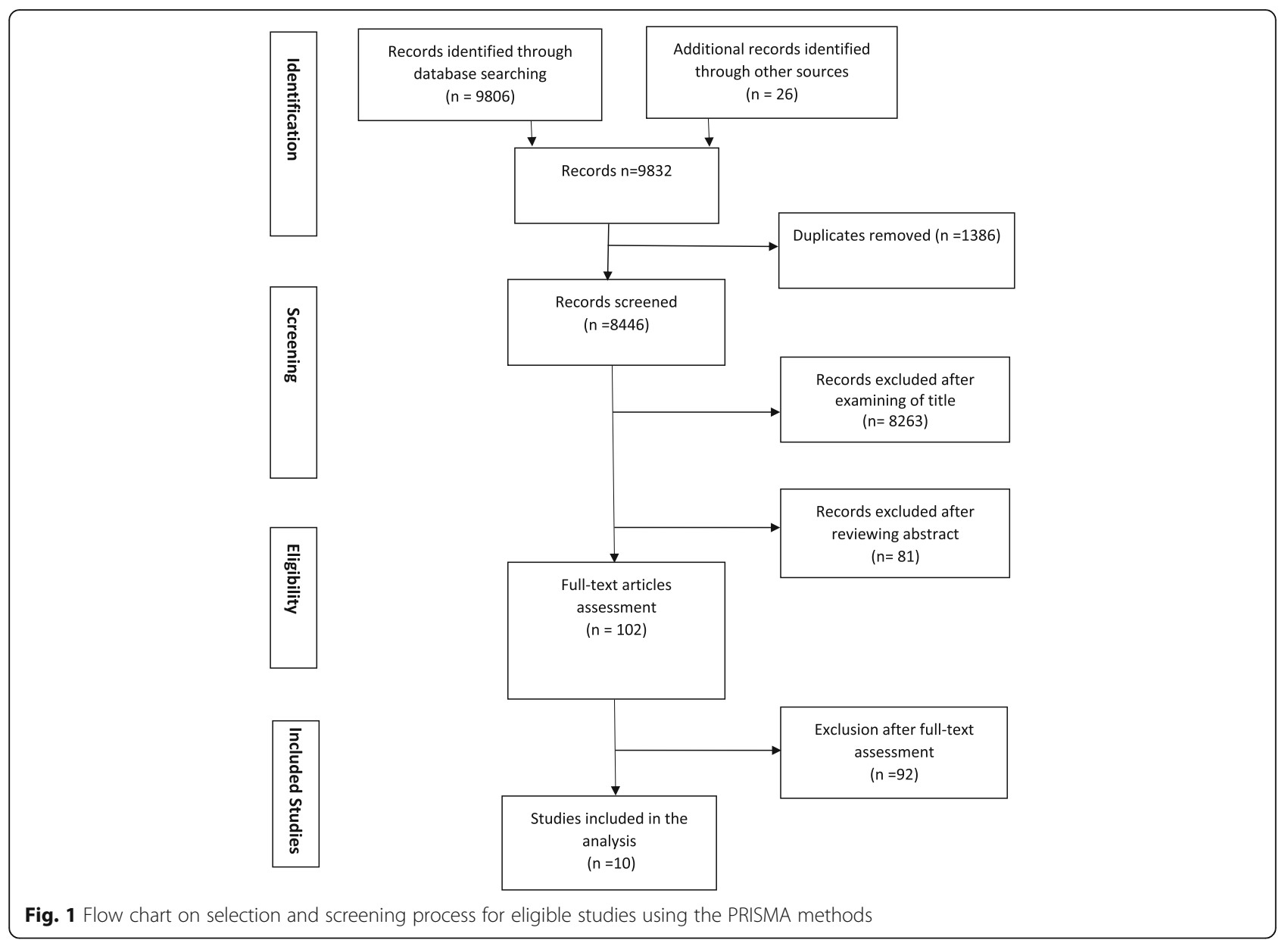

\section{Quality assessment}

The overall quality of the quantitative studies was moderate given that certain methodological limitations were found in the eligible studies. Only one study described in detail the sampling of study subjects and employed sampling techniques to minimise selection bias [69]. However, the rest of the cross sectional studies did not include substantive information on sampling techniques used to minimise selection bias. Three of the eligible studies reported high response rates of 91 [63], 92 [67] and 95.8\% [66]. Conversely, low response rates of 13 [64] and 26\% [69] were also noted. Details of quality of reporting evaluations are presented in Table 4.

On the other hand, none one of the eligible qualitative studies reported on theoretical or philosophical bases for methodological choice, limiting the ability to situate and assess methodological relevance. However, there was a common approach to the reporting of specific data collection and the analysis process. However, none of the studies reported on how the philosophical paradigm influenced data analysis and interpretation. All qualitative studies adequately described how interviews were conducted, although no assessment data trustworthiness through triangulation or member checking was reported. Finally, two of the qualitative studies $[48,62]$ addressed the issue of reflexivity, that is, potential reporting bias related to the researcher's professional background or areas of interest.

\section{Synthesis of results \\ Summary of evidence: main barriers and enablers to evidence uptake}

Overall, four studies reported on both barriers and enablers to uptake of evidence-based acute stroke therapies $[61,62,66,69]$ whilst five reported on only barriers to evidence uptake $[60,63,65,67,67]$ and one study had an explicit focus on enablers to uptake of evidencebased care for acute stroke [63]. Despite some studies reporting on both barriers and enablers, studies which focused only on barriers often made reference to or inferred enablers as the opposite of the barriers, an approach that has been adopted in the present review. Thus, barriers and enablers were analysed and discussed collectively. As reported below, Table 3 shows the 
Table 3 Barriers and enablers to evidence-based acute stroke care

\begin{tabular}{ll}
\hline Domain of barriers and enablers & Frequency (\%) \\
\hline Guideline factors & $16(10.38 \%)$ \\
Individual health professionals & $39(25.32 \%)$ \\
Patient factors & $15(9.74 \%)$ \\
Professional interactions & $10(6.49 \%)$ \\
Incentives and resources & $17(11.03 \%)$ \\
Capacity for organisational change & $57(37.01 \%)$ \\
Social, political and legal factors & $0(0.0 \%)$ \\
\hline
\end{tabular}

Note: The weighted frequency was calculated based on the number of times a particular barrier or enabler was reported in the eligible studies

distribution and weighted frequency of each barrier which provides information on the potential significance of each barrier and enabler to the uptake of the four recommended acute stroke care interventions.

\section{Capacity for organisational change}

This category of barriers/enablers was the most highly cited by participants in all the eligible studies. According to the health professionals, the use of evidence-based care could be challenged by lack of institutional support [61]. They further highlighted limited health staff capacity especially lack of a stroke nurse or specialist [60, $62,65]$ and inadequate funding opportunities for staff professional development $[62,64]$. For example, participants reported that some hospitals were unable to provide or formalise acute stroke care guidelines to facilitate health staff use of evidence-based therapies $[60,69]$. Additionally, instances were cited where there was limited or no executive support for professional development or upgrading to deliver current therapies for acute stroke according to best scientific evidence [62]. Of the varied barriers reported under this category, workload demands were also commonly cited as a key hindrance to the implementation of evidence-based acute stroke care [60, 62, 67]. In one study [69], 71\% of participants indicated lack of protocols and pathways. The study by Van der Weijden et al. identified organisational level barriers as the most significant barriers to uptake of evidencebased practice [68].

\section{Individual health professionals}

Individual health professionals' factors were reported by participants as important barriers/enablers from the eligible studies. This domain of barriers was found in nine included studies [60-65, 67-69]. In the views of most participants, uptake of evidence-based interventions such as thrombolytic therapy is slow or not happening due to health professional's lack of awareness of a particular intervention $[60,61,68,69]$, lack of skills or self-efficacy to apply the intervention $[60,61,68]$ or low motivation to implement an evidence-based therapy [60]. For example, in one study, 50\% of participants indicated their lack of knowledge on the use of thrombolytic therapy hampered uptake in their routine clinical practice [60]. They also outlined barriers such as oldfashioned views about some specific acute stroke therapies [61]. Further, one study [63] reported that some neurologists disapprove of the use of thrombolytic therapy, which was agreed by (33\%) of respondents.

\section{Resources and incentives}

This was another major domain of barriers or enablers to evidence uptake for acute stroke care. A total of eight of ten eligible studies identified resources and incentives related barriers/enablers as crucial to evidence uptake [60-63, 65-68]. Some of the common barriers/enablers comprised limited physical space to establish stroke units [66], lack of CT scans [63], lack of financial resources [61, 62, 66, 68], limited time [61, 66], limited stroke beds $[62,66]$ and limited staff capacity [61-63, $66,68]$. These factors were common in both qualitative and quantitative studies in this review.

\section{Guidelines factors}

The present review has shown the nature and characteristics of specific evidence-based therapies for acute stroke could influence their levels of uptake. Nine of ten eligible studies reported barriers related to the characteristics or the nature of evidence related to the stroke intervention or guidelines [60-63, 65-69]. Views related more to health professionals' misconceptions about the level of effectiveness of some acute stroke care therapies such as thrombolysis. For example, despite evidence that the benefits of thrombolysis outweigh potential associated side effects, participants expressed doubts in the effectiveness of this therapy because they were concerned about severe bleeding and other complications. In one study [69], 73\% of respondents indicated risk of symptomatic intracerebral haemorrhage as a key barrier to administering thrombolysis. In another study [63], $33 \%$ of the participants expressed uncertainty about the evidence of using thrombolytic therapy for acute ischemic stroke and recommended the need for further studies for definitive evidence of its efficacy before they would use it for patient care. Disagreement on the recommended dosage for aspirin was also highlighted by participants in one study [68].

\section{Patient factors}

Within this domain of barriers and enablers, six studies highlighted factors such as late arrival to seek care, patients' or relatives' lack of awareness of early stroke symptoms or patients' decision for other acute care interventions outside the standardised recommendation 
Table 4 Critical appraisal of eligible studies

Appraisal questions for survey

1 Did the study address a clearly focused question/issue?

2 Is the research method (study design) appropriate for answering the research question?

3 Is the method of selection of the subjects (employees, U teams, divisions, organisations) clearly described?

4 Could the way the sample was obtained introduce N (selection) bias?

5 Was the sample of subjects representative with regard N to the population to which the findings will be referred?

6 Was the sample size based on pre-study considerations of statistical power?

7 Was a satisfactory response rate achieved?

8 Are the measurements (questionnaires) likely to be valid and reliable?

9 Was the statistical significance assessed?

10 Are confidence intervals given for the main results?

11 Could there be confounding factors that haven't been accounted for?

12 Can the results be applied to your organisation?

Yes (Y), Can't Tell (U) and NO (N)

Critical Appraisal Questions for Qualitative Studies

1 Is there a congruity between the stated philosophical perspective and the research methodology?

2 Is there a congruity between the research methodology $Y$ and the research question or objectives?

3 Is there a congruity between the research methodology $Y$ and the methods used to collect the data?

4 is there a congruity between the research methodology $Y$ and the representation and analysis of data?

5 Is there a congruity between the research methodology $Y$ and the interpretation of results?

6 Is there a statement locating the researcher culturally or theoretically?

7 Is the influence of the researcher on the research and vice versa addressed?

8 Are participants, and their voices, adequately represented?

9 Is the research ethical according to current criteria or, for recent studies, is there evidence of ethical approval by an appropriate body?

10 Do the conclusions drawn in the research report flow $Y$ from the analysis, or interpretation, of the data?

Yes (Y) No (N) Unclear (U) Not Applicable (NA) $\begin{array}{lllllll}\text { O'Rourke } & \text { William } & \text { Van Der Weijden } & \text { Grady } & \text { Hargis } & \text { Chan } & \text { Slot } \\ \text { (2013) [66] } & \text { (2013) [69] } & \text { (2004) [67] } & \text { (2014) [64] } & \text { (2015) [65] } & \text { (2005) [63] } & \text { (2009) }\end{array}$

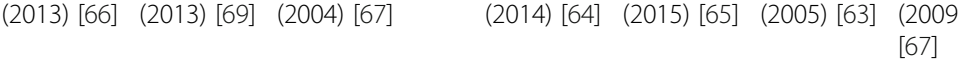

\begin{tabular}{|c|c|c|c|c|c|c|}
\hline Y & Y & $Y$ & Y & $Y$ & $Y$ & Y \\
\hline Y & Y & $Y$ & $Y$ & $Y$ & $Y$ & Y \\
\hline U & Y & $Y$ & $Y$ & $Y$ & $\mathrm{~N}$ & Y \\
\hline$N$ & $\mathrm{~N}$ & $N$ & $\mathrm{~N}$ & $U$ & $\mathrm{~N}$ & $\mathrm{~N}$ \\
\hline$N$ & $N$ & $\mathrm{~N}$ & $Y$ & $N$ & $\mathrm{~N}$ & Y \\
\hline$N$ & $Y$ & $Y$ & $N$ & $\mathrm{~N}$ & $\mathrm{~N}$ & $\mathrm{~N}$ \\
\hline Y & $\mathrm{N}$ & $N$ & $N$ & $Y$ & $Y$ & $Y$ \\
\hline$N$ & $Y$ & $N$ & $Y$ & $N$ & $\mathrm{~N}$ & $\mathrm{~N}$ \\
\hline$N$ & $\mathrm{~N}$ & $N$ & $Y$ & $N$ & $N$ & $\mathrm{~N}$ \\
\hline$N$ & $\mathrm{~N}$ & $Y$ & $\mathrm{~N}$ & $N$ & $\mathrm{~N}$ & $\mathrm{~N}$ \\
\hline$N$ & $\mathrm{~N}$ & $N$ & $N$ & $N$ & $\mathrm{~N}$ & $U$ \\
\hline$N$ & $U$ & $U$ & N & $N$ & $N$ & $Y$ \\
\hline
\end{tabular}

Meurer Purvis Stecksén (2013)

(2011) [60] (2014) [62] [61]

$\mathrm{N} \quad \mathrm{N} \quad \mathrm{N}$

$\begin{array}{lll} & & \\ Y & Y\end{array}$

$Y$
$Y$$\quad \mathrm{Y}$

Y $\quad$ Y $\quad$ Y

$\begin{array}{lll}Y & Y & Y\end{array}$

Y $\quad$ Y $\quad$ N

U $\quad$ Y $\quad U$

$\begin{array}{lll}Y & \text { Y }\end{array}$

Y $\quad Y$
$[60,62,66,67-69]$. The most frequently reported patient-related barrier was patients' late arrival in emergency departments to receive thrombolysis. For example, one study [69] reported that $91 \%$ of respondents indicated patients' late arrival for acute care as the major barrier. Another study ranked delayed patient presentation for care as the major barrier to the use of thrombolytic therapy [65] due to the patients' failure to recognise stroke symptoms. Another key barrier was patients' preference for the non-use of thrombolysis as a therapeutic option due to perceived side-effects of this treatment option [67]. 


\section{Professional interactions}

The uptake of evidence-based care for acute stroke can also be influenced by the form and nature of interactions among health professionals, especially engagement with clinical leaders. Five studies showed evidence of this domain of barriers/enablers [61, 62, 64-66]. The present review found this as among the least described barriers/ enablers in the included studies. Barriers suggested by participants included: inadequate communication especially among clinical staff [65], lack of clinical leadership or support from senior clinicians [62]. As an example, Hargis et al. reported that $14 \%$ of respondents cited poor communication between emergency department staff, and the neurology team affected the use of thrombolytic therapy [65].

\section{Discussion}

This review aimed to explore the main barriers/enablers underlying adoption of evidence-based therapies for treatment and management of acute stroke. To date, prior studies have focused largely on barriers or enablers to generalised acute stroke guidelines or thrombolysis specifically. This review addressed a knowledge gap on the main barriers or enablers to the uptake of the four recommended evidence-based therapies/service for acute stroke, namely, stroke unit care, thrombolytic therapy, aspirin and decompressive surgery.

The specific innovations of this review are its primary focus on the four recommended evidence-based therapies for acute stroke care and the inclusion of both quantitative and qualitative study designs, both of which add depth to the analysis. Although this review was limited to ten eligible studies, there seems to be a saturation of potential determinants given the commonality and recurrence of barriers and enablers revealed between studies. There was also a significant overlap in the reported barriers or enablers, although these characterisations differed between health professionals. Findings from prior reviews on barriers to thrombolysis uptake [41, 44, $45,70]$, other studies on the barriers and enablers to triaging, treatment and patients' transfer in emergency departments (ED) [48] and adherence to general stroke clinical guidelines [40], corroborated with majority of the barriers/enablers identified in this review.

On the most important barriers or enablers from the present review, organisational context or structural level factors were the most cited barriers or enablers to uptake of evidence-based care for acute stroke by health professionals. This finding substantiates the results of earlier works $[9,41,70]$. Given the importance attached to this category of barriers and as reflected in earlier works, a greater effort to address these barriers should be prioritise by health managers and planners for optimal uptake of evidence-based practice. Further, consistent with the literature $[9,40,41,45,71]$, the barriers related to the individual health professional and guideline level barriers, availability of adequate health resources and medical facilities were also predominant in this review.

The barriers/enablers associated with social, political and legal factors were not reported by any of the eligible studies, thus leaving a gap in our understanding of whether such thematic barriers or enablers play any important role in evidence-based care uptake. It is plausible that their influence on evidence-uptake is negligible and may not warrant immediate attention of health policymakers and health managers. The absence of evidence for this domain of barriers/enablers in this review was also evident in the checklist employed to contextualise the discussion in this review. In that review [55], which promulgated the checklist, this particular domain attracted the least eligible studies.

Importantly, the eligible studies were all conducted in high-income countries and so the findings may not be directly relevant to those in low-middle income countries. The inadequacy of medical facilities, limited health staff capacity and other health resource constraints characterised in low- and middle-income countries may emerge as the most important barrier since health systems in these contexts always have fewer resources overall compared to high-income countries.

This review has also underscored the need for increased attention on patient level barriers. Specifically, patients' late arrival in ED settings for care because of lack of recognition of early stroke symptoms was notable. To address the low awareness or lack of early recognition of stroke symptoms, we recommend the need for increased public health campaigns and research emphasising the urgent need to seek care at stroke symptom onset, as highlighted by the 'time is brain' research study [72] and the 'FAST' stroke awareness campaign messages in the UK $[73,74]$. The UK FAST stroke awareness campaign strategy could be a unique exemplar for low- and middleincome countries where evidence [75-77] suggest low awareness of stroke symptoms is a major obstacle to care. With the exception of thrombolytic therapy, the barriers or enablers on the remaining three evidence-based recommendations were less explored. No studies explored decompression surgery, although an earlier review suggested patient level barriers as more essential [45]. Other researchers have cited limited access to computed tomographic $(\mathrm{CT})$ brain scans in low-middle income as the most important factors to address to improve uptake of aspirin therapy [78].

\section{Implications}

The analysis from this review may inform the circumstances in which health professionals are able to provide 
evidence-based care for acute stroke patients. Despite the increased scholarship and policy recommendations for this, the reported barriers or enablers persist, consequently depriving patients of sound and effective therapies. Given that previous evidence suggest, overall, a significant number of patients receive clinical care without sound scientific evidence [2, 10, 79], these findings have the potential to contribute to present efforts aimed at ensuring stroke patients receive effective care.

Increasingly, reports of the rising incidence and mortality rates from stroke in low- and middle-income countries continue to attract the attention of global health authorities. Nonetheless, studies thus far have indicated a low uptake of evidence-based care for acute stroke in Africa and other low/middle income regions $[35,36]$. However, no eligible studies were found in lowand middle-income countries to improve understanding about the factors accounting for this apparent gap. It is essential to explore the barriers or enablers in the context of Africa and other low- and middle-income regions to develop context-specific interventions to enhance uptake of evidence-based care for acute stroke.

Various health professionals play major primary roles as acute caregivers and consequently have unique challenges that deserve attention in future studies since this review was unable to separate determinants according to specific health professionals. Future research should endeavour to explore the barriers or enablers unique to stroke specialists, medical doctors, nurses and allied health staff. As emphasised earlier, identifying the views of stroke patients and carers on the barriers and enablers to stroke care should be part of future research efforts.

\section{Strengths and limitations}

An important strength of this review is its primary focus on the four recommended evidence-based care interventions. The inclusion of both quantitative and qualitative study designs further adds to the analytical breadth and depth of this review. Nonetheless, this review has some limitations. First, we acknowledge that since this study was limited to studies published in English language, there remains a possibility other relevant studies and insights from LMIC were missed. Also, the screening process for eligible studies was conducted by a single author, and this may have affected the accuracy, reliability and transparency of the process. Additionally, the search for relevant studies was limited to only peer-reviewed journals thus potentially relevant theses, conference presentations and book chapters were excluded. Although, the reasons for the lack of studies from low- and middle-income countries remains unclear, this could be explained by the prevailing situation of limited international literature on the uptake of evidence-based acute stroke care interventions from such settings.
The limited number of eligible studies made it impossible to draw definitive conclusions about the primary barriers or enablers to evidence uptake for acute stroke care. Also, although the present study attempted to rank the importance of the barriers and enablers based on their weighted frequencies, this is not optimal. This field is less developed with currently no time-tested approaches to qualitatively rate the importance of such drivers to change in healthcare. Approaches such as the GRADE-CERQual framework to measure the confidence of synthesised evidence [80] could be explored in similar reviews in future. As we used a pre-designed taxonomy of barriers and enablers to contextualise our findings, it is possible other relevant barriers and enablers considered unfit to the framework were inadvertently missed out.

\section{Conclusions}

The reported barriers or enablers mapped well with the previously proposed taxonomy of barriers or enablers. Our findings are consistent with previous studies $[9,40]$ where lack of adherence to or inadequate use of evidencebased care was attributed to organisational level factors, professionals' lack of awareness and familiarity to a particular evidence-based care, financial constraints, lack of confidence in a particular therapy, fear of adverse effects, personal beliefs, patient delays, lack of time to implement evidence-based treatment guidelines and preferences or values about the use of evidence-based care.

Despite considerable effective therapeutic options for acute stroke care, poor understanding of barriers or enablers and lack of a clear evidence-based health policy to ensure their uptake render such therapeutic services underutilised. In light of this, efforts by health managers and policy-makers to formulate context-specific policies and design interventions to enhance uptake of evidencebased care should be informed by these barriers and enablers. Following this review, we are also proposing research studies be conducted in low-middle income countries to enhance our understanding of the key barriers accounting for the currently low uptake levels of evidence-based acute stroke care interventions.

\section{Additional files}

Additional file 1: PRISMA 2009 Checklist. (DOCX $15 \mathrm{~kb}$ )

Additional file 2: Search strategy for MEDLINE. (DOCX 17kb)

Additional file 3: Checklist for Barriers and Enablers. (DOCX 12 kb)

\section{Abbreviations}

AMED: Allied and Complementary Medicine Database; CINAHL: Cumulative Index to Nursing and Allied Health Literature; CT: Computed tomographic; ED: Emergency department; FAST: Facial drooping, arm weakness, speech difficulties and time; GRADE-CERQual: Confidence in the Evidence from Reviews of Qualitative Research; ICU: Intensive care unit; MeSH: Medical Subject Heading; NINDS: National Institute of Neurological Disorders and 
Stroke; PRISMA: Preferred Reporting Item for Systematic Reviews and MetaAnalysis; t-PA: Intravenous tissue plasminogen activator

\section{Acknowledgements}

We thank Samantha Fernandez, Liaison Librarian, North Sydney Campus of the Australian Catholic University for her assistance during the development of the search strategy and to Julia Sterman and Anthony Sumah for proofreading the initial draft of the manuscript.

\section{Funding}

The authors declare no funding support for this review.

\section{Availability of data and materials}

The authors declare that the data supporting the study findings are available within the article and Additional files.

\section{Authors' contributions}

LB is responsible for the protocol design, literature search of databases, study selection and data extraction processes. MO, SS, GM, AdGA and JC contributed to the selection and data extraction processes. LB and SS helped in quality appraisal process. LB AdGA and SS are responsible for the classification of the themes. LB wrote the first draft of the manuscript. MO, SS, GM, AdGA and JC reviewed the manuscript for content analysis and discussion. SS, MO, GM, AdGA and JC supervised and coordinated the entire review process. All authors read and approved the final manuscript.

\section{Competing interests}

The authors declare that they have no competing interests.

\section{Consent for publication}

Not applicable.

Ethics approval and consent to participate Not applicable.

\section{Publisher's Note}

Springer Nature remains neutral with regard to jurisdictional claims in published maps and institutional affiliations.

\section{Author details}

${ }^{1}$ Regional Institute for Population Studies, University of Ghana, P.O Box LG96, Legon-Accra, Ghana. ${ }^{2}$ School of Allied Health, Faculty of Health Sciences, Australian Catholic University, Sydney, Australia. ${ }^{3}$ College of Health Sciences, University of Sharjah, Sharjah, United Arab Emirates. ${ }^{4}$ College of Science, Health and Engineering, La Trobe Rural Health School, La Trobe University, Melbourne, Australia. ${ }^{5}$ School of Pharmacy, University of Queensland, Brisbane, Australia.

\section{Received: 10 October 2016 Accepted: 8 May 2017}

\section{Published online: 05 June 2017}

\section{References}

1. Cochrane A. Effectiveness and efficiency: Random reflections on health services. London: Nuffield Provincial Hospitals Trust; 1972.

2. Grol R, Wensing M, Eccles M, Davis D. Improving patient care: the implementation of change in health care: John Wiley \& Sons; 2013.

3. Straus S, Tetroe J, Graham ID. Knowledge translation in health care: moving from evidence to practice: John Wiley \& Sons; 2013.

4. Grimshaw J, Eccles M, Thomas R, MacLennan G, Ramsay C, Fraser C, et al. Toward Evidence-Based Quality Improvement. J Gen Intern Med. 2006; 21(S2):S14-20.

5. World Health Organisation. Research for Universal Health Coverage. Geneva: WHO; 2013

6. Dopson S, Locock L, Gabbay J, Ferlie E, Fitzgerald L. Evidence-based medicine and the implementation gap. Health. 2003:7(3):311-30.

7. Grol R, Grimshaw J. From best evidence to best practice: effective implementation of change in patients' care. Lancet. 2003:362(9391):1225-30.

8. Morris ZS, Wooding S, Grant J. The answer is 17 years, what is the question: understanding time lags in translational research. J R Soc Med. 2011;104(12): 510-20.
9. Cabana MD, Rand CS, Powe NR, Wu AW, Wilson MH, Abboud P-AC, et al. Why don't physicians follow clinical practice guidelines?: A framework for improvement. JAMA. 1999;282(15):1458-65.

10. Grimshaw JM, Eccles MP, Lavis JN, Hill SJ, Squires JE. Knowledge translation of research findings. Implement Sci. 2012;7(1):50.

11. Baker R, Camosso-Stefinovic J, Gillies C, Shaw EJ, Cheater F, Flottorp S, et al. Tailored interventions to overcome identified barriers to change: effects on professional practice and health care outcomes. Cochrane Database Syst Rev. 2010;3(3).

12. Cochrane LJ, Olson CA, Murray S, Dupuis M, Tooman T, Hayes S. Gaps between knowing and doing: understanding and assessing the barriers to optimal health care. J Contin Educ Health Prof. 2007;27(2):94-102.

13. SURE Collaboration. SURE Guides for Preparing and Using Evidence-Based Policy Briefs: 5 Identifying and addressing barriers to implementing policy options. 2011.

14. Harvey G, Kitson A. Translating evidence into healthcare policy and practice: Single versus multi-faceted implementation strategies-is there a simple answer to a complex question? Int J Health Policy Manag. 2015;4(3):123

15. Norrving B. Oxford Textbook of Stroke and Cerebrovascular Disease: Oxford University Press; 2014.

16. World Health Organization. Neurological disorders: public health challenges: World Health Organization; 2006

17. Wright L, Hill KM, Bernhardt J, Lindley R, Ada L, Bajorek BV, et al. Stroke management: updated recommendations for treatment along the care continuum. Intern Med J. 2012;42(5):562-9.

18. Trialists'Collaboration SU. Organised inpatient (stroke unit) care for stroke. Cochrane Database Syst Rev. 2013(4).

19. Langhorne P, Williams BO, Gilchrist W, Howie K. Do stroke units save lives? Lancet. 1993;342(8868):395-8.

20. Wardlaw JM MV, Berge E, del Zoppo GJ,. Thrombolysis for acute ischaemic stroke. Cochrane Database of Syst Rev 2014. 2014;Issue 7.

21. Hacke W, Kaste M, Bluhmki E, Brozman M, Dávalos A, Guidetti D, et al. Thrombolysis with alteplase 3 to 4.5 hours after acute ischemic stroke. N Engl J Med. 2008;359(13):1317-29.

22. NINDS. The National Institute of Neurological Disorders and Stroke Study Group. N Engl J Med. 1995:333:1581-7.

23. Sandercock $P$, Counsell $C$, Tseng M, Cecconi E. Oral antiplatelet therapy for acute ischaemic stroke (Review). Cochrane Database Syst Rev. 2014(3).

24. Collaboration AT. Collaborative overview of randomised trials of antiplatelet therapy Prevention of death, myocardial infarction, and stroke by prolonged antiplatelet therapy in various categories of patients. BMJ. 1994;308(6921):81-106.

25. Group ISTC. The International Stroke Trial (IST): a randomised trial of aspirin, subcutaneous heparin, both, or neither among 19435 patients with acute ischaemic stroke. Lancet. 1997;349(9065):1569-81.

26. Vahedi K, Hofmeijer J, Juettler E, Vicaut E, George B, Algra A, et al. Early decompressive surgery in malignant infarction of the middle cerebral artery: a pooled analysis of three randomised controlled trials. Lancet Neurol. 2007; 6(3):215-22

27. Cruz-Flores S, Berge E, Whittle IR. Surgical decompression for cerebral oedema in acute ischaemic stroke. The Cochrane Library. 2012.

28. National Institute for Health and Clinical Excellence. Diagnosis and initial management of acute stroke and transient ischaemic attack (TIA). London: Nation Collaborating Centre for Chronic Conditions; 2008. Report No.: 1860163394

29. National Stroke Foundation. Clinical guidelines for stroke management 2010. Victoria: National Stroke Foundation; 2010.

30. Casaubon LK, Boulanger JM, Blacquiere D, Boucher S, Brown K, Goddard T, et al. Canadian stroke best practice recommendations: hyperacute stroke care guidelines, update 2015. Int J Stroke. 2015;10(6):924-40.

31. Jauch EC, Saver JL, Adams HP, Bruno A, Demaerschalk BM, Khatri P, et al Guidelines for the early management of patients with acute ischemic stroke a guideline for healthcare professionals from the American Heart Association/American Stroke Association. Stroke. 2013;44(3):870-947.

32. Steiner T, Al-Shahi Salman R, Beer R, Christensen H, Cordonnier C, Csiba L, et al. European Stroke Organisation (ESO) guidelines for the management of spontaneous intracerebral hemorrhage. Int J Stroke. 2014;9(7):840-55.

33. Intercollegiate Stroke Working Party. National clinical guideline for stroke. 4th ed. London: Royal College of Physicians; 2012

34. Hemphill JC, Greenberg SM, Anderson CS, Becker K, Bendok BR, Cushman $M$, et al. Guidelines for the management of spontaneous intracerebral hemorrhage. Stroke. 2015;46(7):2032-60. 
35. Berkowitz AL, Mittal MK, McLane HC, Shen GC, Muralidharan R, Lyons JL, et al. Worldwide reported use of IV tissue plasminogen activator for acute ischemic stroke. Int J Stroke. 2014;9(3):349-55.

36. Langhorne $P$, de Villiers L, Pandian JD. Applicability of stroke-unit care to low-income and middle-income countries. Lancet Neurol. 2012;11(4):341-8.

37. Norrving B, Kissela B. The global burden of stroke and need for a continuum of care. Neurology. 2013;80(3 Suppl 2):S5-12.

38. Baatiema L, Otim M, Mnatzaganian G, Aikins AD-G, Coombes J, Somerset S. Towards best practice in acute stroke care in Ghana: a survey of hospital services. BMC Health Serv Res. 2017;17(1):108.

39. Dale S, Levi C, Ward J, Grimshaw JM, Jammali-Blasi A, D'Este C, et al. Barriers and Enablers to Implementing Clinical Treatment Protocols for Fever, Hyperglycaemia, and Swallowing Dysfunction in the Quality in Acute Stroke Care (QASC) Project-A Mixed Methods Study. Worldviews Evid-Based Nurs. 2015;12(1):41-50.

40. Donnellan C, Sweetman S, Shelley E. Health professionals' adherence to stroke clinical guidelines: a review of the literature. Health Policy. 2013;111(3):245-63.

41. Kwan J, Hand P, Sandercock P. A systematic review of barriers to delivery of thrombolysis for acute stroke. Age Ageing. 2004;33(2):116-21.

42. Sandercock P, Berge E, Dennis M, Forbes J, Hand P, Kwan J, et al. A systematic review of the effectiveness, cost-effectiveness and barriers to implementation of thrombolytic and neuroprotective therapy for acute ischaemic stroke in the NHS. Health Technol Assess (Winchester, England). 2001;6(26):1-112.

43. Eissa A, Krass I, Bajorek B. Barriers to the utilization of thrombolysis for acute ischaemic stroke. J Clin Pharm Ther. 2012;37(4):399-409.

44. Johnson M, Bakas T. A review of barriers to thrombolytic therapy: Implications for nursing care in the emergency department. J Neurosci Nurs. 2010;42(2):88-94

45. Sandercock P, Berge E, Dennis M, Forbes J, Hand P, Kwan J, et al. A systematic review of the effectiveness, cost-effectiveness and barriers to implementation of thrombolytic and neuroprotective therapy for acute ischaemic stroke in the NHS. Health Technol Assess. 2002;6(26):1-112.

46. Baatiema L, De-Graft Aikins A, Sav A, Mnatzaganian G, Chan CKY. Somerset S. Barriers to evidence-based acute stroke care in Ghana: A qualitative study on the perspectives of stroke care professionals. BMJ Open. 2017;7(4): e015385. doi:10.1136/bmjopen-2016-015385.

47. Glasziou P, Straus S, Brownlee S, Trevena L, Dans L, Guyatt G, ... \& Saini V. Evidence for underuse of effective medical services around the world. The Lancet. 2017. doi.org/10.1016/S0140-6736(16)30946-1

48. Craig LE, McInnes E, Taylor N, Grimley R, Cadilhac DA, Considine J, et al. Identifying the barriers and enablers for a triage, treatment, and transfer clinical intervention to manage acute stroke patients in the emergency department: a systematic review using the theoretical domains framework (TDF). Implement Sci. 2016;11(1):157.

49. Feigin VL, Lawes CM, Bennett DA, Barker-Collo SL, Parag V. Worldwide stroke incidence and early case fatality reported in 56 population-based studies: a systematic review. Lancet Neurol. 2009;8(4):355-69.

50. Johnston SC, Mendis S, Mathers CD. Global variation in stroke burden and mortality: estimates from monitoring, surveillance, and modelling. Lancet Neurol. 2009;8(4):345-54.

51. Feigin VL, Roth GA, Naghavi M, Parmar P, Krishnamurthi R, Chugh S, et al. Global burden of stroke and risk factors in 188 countries, during 1990-2013: a systematic analysis for the Global Burden of Disease Study 2013. Lancet Neurol. 2016.

52. Moher D, Liberati A, Tetzlaff J, Altman DG. Preferred reporting items for systematic reviews and meta-analyses: the PRISMA statement. Ann Intern Med. 2009;151(4):264-9.

53. Dopson S, Fitzgerald L. Knowledge to action?: evidence-based health care in context. Oxford: Oxford University Press; 2005

54. Wensing M, Oxman A, Baker R, Godycki-Cwirko M, Flottorp S, Szecsenyi J, et al. Tailored implementation for chronic diseases (TICD): a project protocol. Implement Sci. 2011;6(1):103.

55. Flottorp SA, Oxman AD, Krause J, Musila NR, Wensing M, Godycki-Cwirko M, et al. A checklist for identifying determinants of practice: a systematic review and synthesis of frameworks and taxonomies of factors that prevent or enable improvements in healthcare professional practice. Implement Sci. 2013;8(1):35

56. Joanna Briggs Institute. Systematic Review Resource Package. Adelaide: The Joanna Briggs Institute; 2015
57. Centre for Evidence Based Management. Critical appraisal of a survey UK2014 [Available from:http://www.cebma.org/wp-content/uploads/CriticalAppraisal-Questions-for-a-Survey.pdf.

58. Dixon-Woods M, Bonas S, Booth A, Jones DR, Miller T, Sutton AJ, et al. How can systematic reviews incorporate qualitative research? A critical perspective. Qual Res. 2006;6(1):27-44.

59. Atkins S, Lewin S, Smith H, Engel M, Fretheim A, Volmink J. Conducting a meta-ethnography of qualitative literature: lessons learnt. BMC Med Res Methodol. 2008;8(1):21.

60. Meurer WJ, Majersik JJ, Frederiksen SM, Kade AM, Sandretto AM, Scott PA. Provider perceptions of barriers to the emergency use of tPA for acute ischemic stroke: a qualitative study. BMC Emerg Med. 2011;11(1):5.

61. Stecksén A, Lundman B, Eriksson M, Glader E-L, Asplund K. Implementing Thrombolytic Guidelines in Stroke Care Perceived Facilitators and Barriers. Qual Health Res. 2013:1049732313514137.

62. Purvis T, Moss K, Denisenko S, Bladin C, Cadilhac DA. Implementation of evidence-based stroke care: enablers, barriers, and the role of facilitators. J Multidiscip Healthc. 2014;7:389.

63. Chan Y-F, Kwiatkowski TG, Rella JG, Rennie WP, Kwon RK, Silverman RA. Tissue plasminogen activator for acute ischemic stroke: a New York city emergency medicine perspective. J Emerg Med. 2005;29(4):405-8.

64. Grady A, Bryant J, Carey M, Paul C, Sanson-Fisher R. Enablers of the Implementation of Tissue Plasminogen Activator in Acute Stroke Care: A Cross-Sectional Survey. PLoS One. 2014;9(12):e114778.

65. Hargis M, Shah JN, Mazabob J, Rao CV, Suarez JI, Bershad EM. Barriers to administering intravenous tissue plasminogen activator (tPA) for acute ischemic stroke in the emergency department: A cross-sectional survey of stroke centers. Clin Neurol Neurosurg. 2015;135:79-84.

66. O'Rourke F, Chan DKY, Chan DL, Ding XM. Preferences, barriers and facilitators for establishing comprehensive stroke units: a multidisciplinary survey. Aust Health Rev. 2013;37(3):318-23.

67. Slot KB, Murray V, Boysen G, Berge E. Thrombolytic treatment for stroke in the Scandinavian countries. Acta Neurol Scand. 2009:120(4):270-6.

68. Van Der Weijden T, Hooi JD, Grol R, Limburg M. A multidisciplinary guideline for the acute phase of stroke: barriers perceived by Dutch neurologists. J Eval Clin Pract. 2004;10(2):241-6.

69. Williams JM, Jude MR, Levi CR. Recombinant tissue plasminogen activator (rt-PA) utilisation by rural clinicians in acute ischaemic stroke: A survey of barriers and enablers. Aust J Rural Health. 2013;21(5):262-7.

70. Ehlers L, Jensen LG, Bech MA, Andersen G, Kjølby M. Organisational barriers to thrombolysis treatment of acute ischaemic stroke. Curr Med Res Opin. 2007;23(11):2833-9.

71. Donnellan C, Sweetman S, Shelley E. Implementing clinical guidelines in stroke: a qualitative study of perceived facilitators and barriers. Health Policy. 2013;111(3):234-44.

72. Saver JL. Time is brain — quantified. Stroke. 2006:37(1):263-6.

73. Dombrowski SU, Mackintosh JE, Sniehotta FF, Araujo-Soares V, Rodgers H, Thomson RG, et al. The impact of the UK 'Act FAST'stroke awareness campaign: content analysis of patients, witness and primary care clinicians' perceptions. BMC Public Health. 2013;13(1):915.

74. Mellon L, Hickey A, Doyle F, Dolan E, Williams D. Can a media campaign change health service use in a population with stroke symptoms? Examination of the first Irish stroke awareness campaign. Emerg Med J. 2014:31(7):536-40.

75. Donkor E, Owolabi M, Bampoh P, Aspelund T, Gudnason V. Community awareness of stroke in Accra, Ghana. BMC Public Health. 2014;14(1):196.

76. Mensah GA. Epidemiology of stroke and high blood pressure in Africa. Heart. 2008;94(6):697-705.

77. Brainin $M$, Teuschl $Y$, Kalra L. Acute treatment and long-term management of stroke in developing countries. Lancet Neurol. 2007;6(6):553-61.

78. Thrift AG, de los Rios F. Expanding antiplatelet use for patients with stroke The case for stroke of unknown type. Neurology. 2014;83(9):778-9.

79. Schuster MA, McGlynn EA, Brook RH. How good is the quality of health care in the United States? Milbank Q. 1998;76(4):517-63.

80. Lewin S, Glenton C, Munthe-Kaas H, Carlsen B, Colvin CJ, Gülmezoglu M, et al. Using qualitative evidence in decision making for health and social interventions: an approach to assess confidence in findings from qualitative evidence syntheses (GRADE-CERQual). PLoS Med. 2015;12(10):e1001895. 